\title{
The Grad-Shafranov Reconstruction of Toroidal Magnetic Flux Ropes: First Applications
}

\author{
Qiang $\mathrm{Hu}^{1}$ (D) . Mark G. Linton ${ }^{2}$. \\ Brian E. Wood ${ }^{2}$. Pete Riley ${ }^{3}$. \\ Teresa Nieves-Chinchilla ${ }^{2,4}$
}

(C) Springer $\bullet \bullet \bullet \bullet$

\begin{abstract}
This article completes and extends a recent study of the Grad-Shafrano (GS) reconstruction in toroidal geometry, as applied to a two and a half dimensional configurations in space plasmas with rotational symmetry. A further application to the benchmark study of an analytic solution to the toroidal GS equation with added noise shows deviations in the reconstructed geometry of the flux rope configuration, characterized by the orientation of the rotation axis, the major radius, and the impact parameter. On the other hand, the physical properties of the flux rope, including the axial field strength, and the toroidal and poloidal magnetic flux, agree between the numerical and exact GS solutions. We also present a real event study of a magnetic cloud flux rope from in situ spacecraft measurements. The devised procedures for toroidal GS reconstruction are successfully executed. Various geometrical and physical parameters are obtained with associated uncertainty estimates. The overall configuration of the flux rope
\end{abstract}

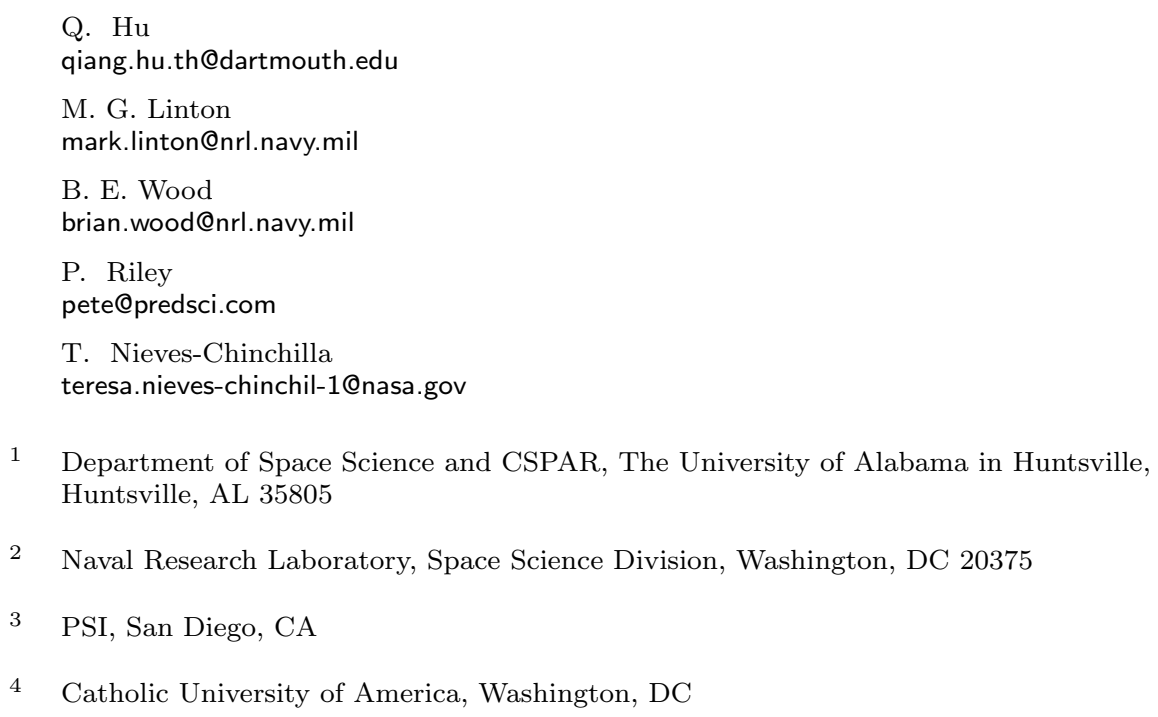

1 Department of Space Science and CSPAR, The University of Alabama in Huntsville, Huntsville, AL 35805

2 Naval Research Laboratory, Space Science Division, Washington, DC 20375

3 PSI, San Diego, CA

4 Catholic University of America, Washington, DC 
from the GS reconstruction is compared with the corresponding morphological reconstruction based on white-light images. The results show overall consistency, but also discrepancy in that the inclination angle of the flux rope central axis with respect to the ecliptic plane differs by about 20-30 degrees in the plane of the sky. We also compare the results with the original straight-cylinder GS reconstruction and discuss our findings.

Keywords: Grad-Shafranov equation; Flux rope, Magnetic; Magnetic Clouds; Magnetic fields, Heliosphere; MHD equilibrium

\section{Introduction}

This article is a continuation of the recently completed study, now in press $\mathrm{CH}$, 2017b, hereafter Paper I), which describes in detail the two-step recipe for the Grad-Shafranov (GS) reconstruction of magnetic flux ropes, or magnetic clouds (MCs), with a toroidal geometry. Under such a geometry, the local configuration of the flux rope in the vicinity of the spacecraft path is approximated by a section of a torus, instead of a straight cylinder. But the symmetry of the structure remains, changing from the translation symmetry of the former straight-cylinder case to the rotational symmetry in the toroidal geometry, so that the central axis of the flux rope in the latter case possesses a finite radius (non-zero curvature).

Under these two-dimensional (2D) or 2.5D configurations with non-vanishing axial magnetic field component, the Grad-Shafranov (GS) equation applies for both configurations, governing the magnetic and plasma structure in magnetohydrostatic equilibrium. The GS reconstruction technique has been developed and applied to reconstruct the $2 \mathrm{D}$ cross section of cylindrical magnetic and plasma configuration, using in situ spacecraft measurements, for the past twenty years. The technique first originated from the application to the magnetopause current sheet crossings (Sonnerup and Guo, 1996, Hau and Sonnerup, 1999). Later it was applied to reconstructing cross sections of magnetic flux ropes, including applications to small-scale flux ropes in the solar wind ( $\mathrm{Hu}$ and Sonnerup, 2001) and to large-scale MCs (Hu and Sonnerup, 2002) for the first time. Additional applications include reconstruction of flux transfer events (FTEs) in Earth's magnetopause, plasmoids in the tail, and even recently to flux ropes in the Martian atmosphere (Hara et al., 2014, 2016). The extensions of the GS reconstruction technique to GS-type applications were also developed, which goes beyond static equilibrium, especially allowing for description of dynamic equilibrium involving significant remaining flow and inertial forces, but remaining in a cylindrical geometry (Hu, 2017a and references therein). Recent extension to a toroidal geometry was fully developed and reported in Paper I, with detailed benchmark studies.

A recent review, commemorating the occasion of twenty year's application of the GS reconstruction method, is presented by $\mathrm{Hu}$ (2017a). That article reviews the history of the development of the technique and summarizes its main applications to recovering various 2.5D space plasma structures, including the magnetopause current sheet, the FTEs, flux ropes in geotail, and magnetic flux 
ropes (and MCs) in the solar wind. In particular, emphasis was put on validation of GS reconstruction results by direct co-spatial multi-spacecraft measurements and indirect quantitative correlation among physical properties between in situ and solar source region analysis, especially through inter comparison of magnetic flux and helicity content for MCs with associated flares and coronal mass ejections (CMEs) (Qiu et al., 2007, Kazachenko et al., 2012 Hu et al., 2014). In addition, field-line twist and length distributions inside MCs were also derived from the GS reconstruction results and were used to assist in interpretation of flux rope configuration and formation through magnetic reconnection processes (Hu, Qiu, and Krucker, 2015, Hu et al., 2014). A qualitative comparison of the GS reconstruction with toroidal geometry to the numerical simulation result of Riley et al. (2004) was also presented in $\mathrm{Hu}(2017 \mathrm{a})$ as an extension to the original GS reconstruction technique with straight-cylinder geometry.

Traditionally, the modeling of magnetic flux rope configurations based on the in situ single spacecraft measurements is performed via least-squares fitting of a specific analytic model to the time-series data (mostly magnetic field vectors). The use of a toroidal flux rope model was developed by Marubashi (1997) and Romashets and Vandas (2003), usually with a circular cross section and a large aspect ratio (the ratio between the major and minor radii of the torus). Hidalgo and colleagues have also developed over the years a sophisticated algorithm for fitting in situ spacecraft measurements with a flux rope model of non-circular cross section, a global configuration conforming to a $3 \mathrm{D}$ rope structure rooted on the Sun, and/or non-field aligned current (e.g. Nieves-Chinchilla et al., 2016 , Hidalgo, 2016, and references therein). The GS reconstruction differs from these fitting based approaches by yielding a completely $2 \mathrm{D}$ cross section as a numerical solution to a more general non-force free state, although fitting is also involved but on one magnetic field component only (the axial field component). Another difference is that the current implementation of the toroidal GS reconstruction does not apply to the situation of a submerged spacecraft path, i.e. a path not exiting into the center hole of the torus, as we illustrated in detail in Paper I. In this situation, one has to resort to the traditional fitting method for a toroidal geometry, as advocated by Marubashi et al. (2015). Some interesting perspectives of in situ flux rope modeling that go beyond static or 2D configurations were also discussed in $\mathrm{Hu}$ (2017a), especially regarding an intrinsically 3D configuration with ribbon-like structures often present in solar prominence observations, resembling a "stellarator" configuration in fusion devices (Freidberg, 2014).

The current article is organized as follows. In the next two sections, we present two case studies: one being a further benchmark study continuing from Paper I, and the other being a real event study observed by the Solar Terrestrial Relations Observatory (STEREO) B spacecraft, following the procedures delineated in Paper I. In particular, we present a comparison of the toroidal GS reconstruction result with the morphological modeling based on white-light images of the real event. Lastly we conclude and summarize our results. 
(a)

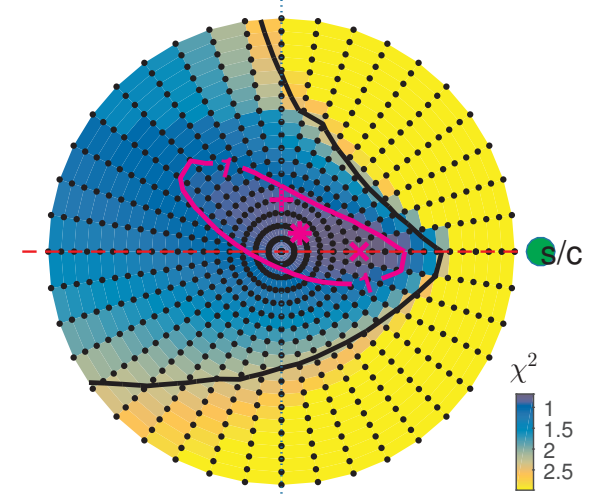

(b)

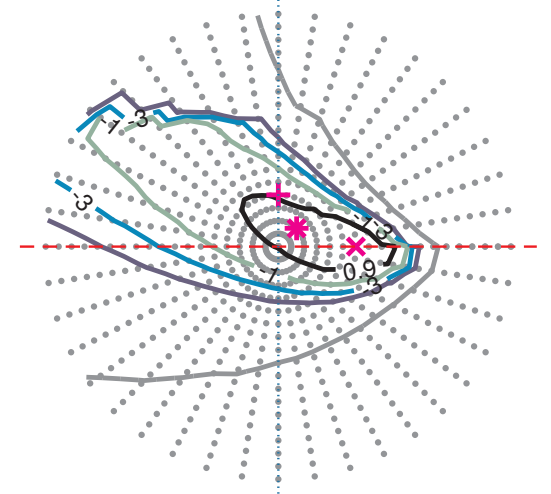

Figure 1. (a) The distribution of reduced $\chi^{2}$ value, as indicated by the colorbar on the $\left(r_{s c}, t\right)$ plane within the circular domain centered on the Sun with a radius of 1 AU. The background dots are the search grid of the intersections of $Z$ axis on that plane. The contours are of levels 1 (magenta), and $1+\sqrt{2}$ (black), respectively. (b) The corresponding contour plot of $\log _{10} Q$ as labeled. The innermost contour is of level $Q=0.9$, and the outermost one is the same as the black one in (a). In each plot, the coordinate system $\left(r_{s c}, t, n\right)$ is centered on the Sun in the middle with $r_{s c}$ pointing radially out to the spacecraft location (green dot in a) along the red dashed line, $t$ pointing upward along the dotted blue line, and $n$ pointing vertically out of the plane at the center. The plus and cross signs mark the true $Z$ axis location and that of $\chi_{\text {min }}^{2}$, respectively. The asterisk sign near the center of the $\chi^{2}=1$ contour denotes the one chosen for the final numerical GS reconstruction.

\section{Application to a Benchmark Case}

We have performed the toroidal GS reconstruction study of the numerical simulation result of Riley et al. (2004), where a flux rope configuration with exact toroidal geometry, but with full MHD and temporal evolution, was generated in the computational domain, covering the inner heliosphere from the Sun to $\sim 1 \mathrm{AU}$. However a quantitative comparison of various physical quantities cannot be made, because the quantitative results of that simulation cannot be recovered due to the long time period elapsed. The qualitative comparison in terms of the orientation, the basic geometry and shape of the flux rope is presented and discussed in $\mathrm{Hu}(2017 \mathrm{a})$.

Here we present a benchmark study that is one step further than the one presented in Paper I, where the benchmark study of an analytic case with general toroidal configuration and noise addition terminated after the determination of the rotation axis $Z$ and the major radius $R=r_{0}$, without the final reconstruction step of applying the GS solver to obtain a solution to the toroidal GS equation. In what follows, we complete this last step and present a quantitative comparison between the exact and numerical solutions, following the procedures given in Paper I, as to be employed when examining a real event.

We refer readers to Paper I for detailed description of the two-step recipe in deriving the main geometrical parameters and the implementation of the GS solver for the toroidal GS equation, using in situ spacecraft data. In short, a trial and error process is carried out to find the optimal orientation of the 

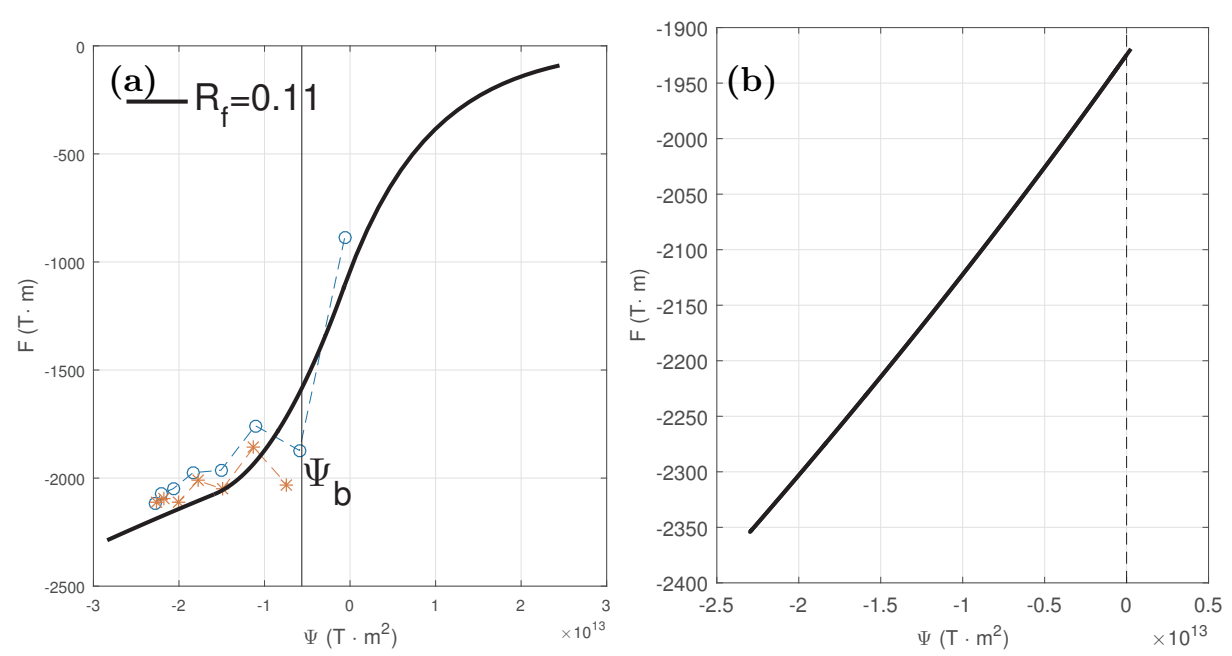

Figure 2. The corresponding measured $F$ versus $\Psi$ data points along the spacecraft path, and the 2nd-order polynomial fitting $F(\Psi)$ (black curve) for (a) the numerical GS solution. A fitting residue $R_{f}$ and a flux rope boundary $\Psi=\Psi_{b}$ are also marked (Hu et al., 2004). The corresponding exact $F(\Psi)$ function is shown in (b).

rotation axis, $Z$, around which the rotational symmetry is satisfied, i.e. in the cylindrical $(R, \phi, Z)$ coordinate system, i.e. $\partial / \partial \phi \approx 0$. The cross sectional plane is $(R, Z)$. In the first step, the orientation of $Z$ axis is determined by fulfilling the requirement that the composite function $F=R B_{\phi}$ be a single-valued function of the magnetic flux function $\Psi$ along the spacecraft path. Then, a $\chi^{2}$ minimization procedure (Press et al., 2007) is performed by minimizing the deviation between the measured and GS model predicted magnetic field components along the spacecraft path, while altering the location of the $Z$ axis over a search grid on the $\left(r_{s c}, t\right)$ plane, as illustrated in Figure 1 a. During this second step, a proper formulation following Press et al. (2007) is adopted with measurement uncertainties assigned to each data point. An optimal location, or the intersection of $Z$ axis with the $\left(r_{s c}, t\right)$ plane is chosen with uncertainty bounds, which in turn yields the major radius $r_{0}$ of the torus. These uncertainty bounds lead to estimates of uncertainty of other associated quantities as to be discussed later. Once the main geometrical parameters, $Z$ and $r_{0}$, are determined by the twostep procedures, the final reconstruction is performed by applying the toroidal GS solver to obtain the cross section of the flux rope in an annular region (Paper I).

Figure 1 shows the determination of the location of the $Z$ axis as the intersection of $Z$ with the $\left(r_{s c}, t\right)$ plane through the $\chi^{2}$ minimization procedures after the first step when an optimal $Z$ axis orientation is chosen from the residue map (see Paper I). The advantage of such an approach with measurement uncertainties is to enable proper $\chi^{2}$ statistics in order to provide an objective assessment of the goodness-of-fit (Press et al., 2007). A reduced $\chi^{2}$ value $\approx 1$ represents the range of uncertainty for the optimal parameter to be determined, i.e. the location of $Z$ 

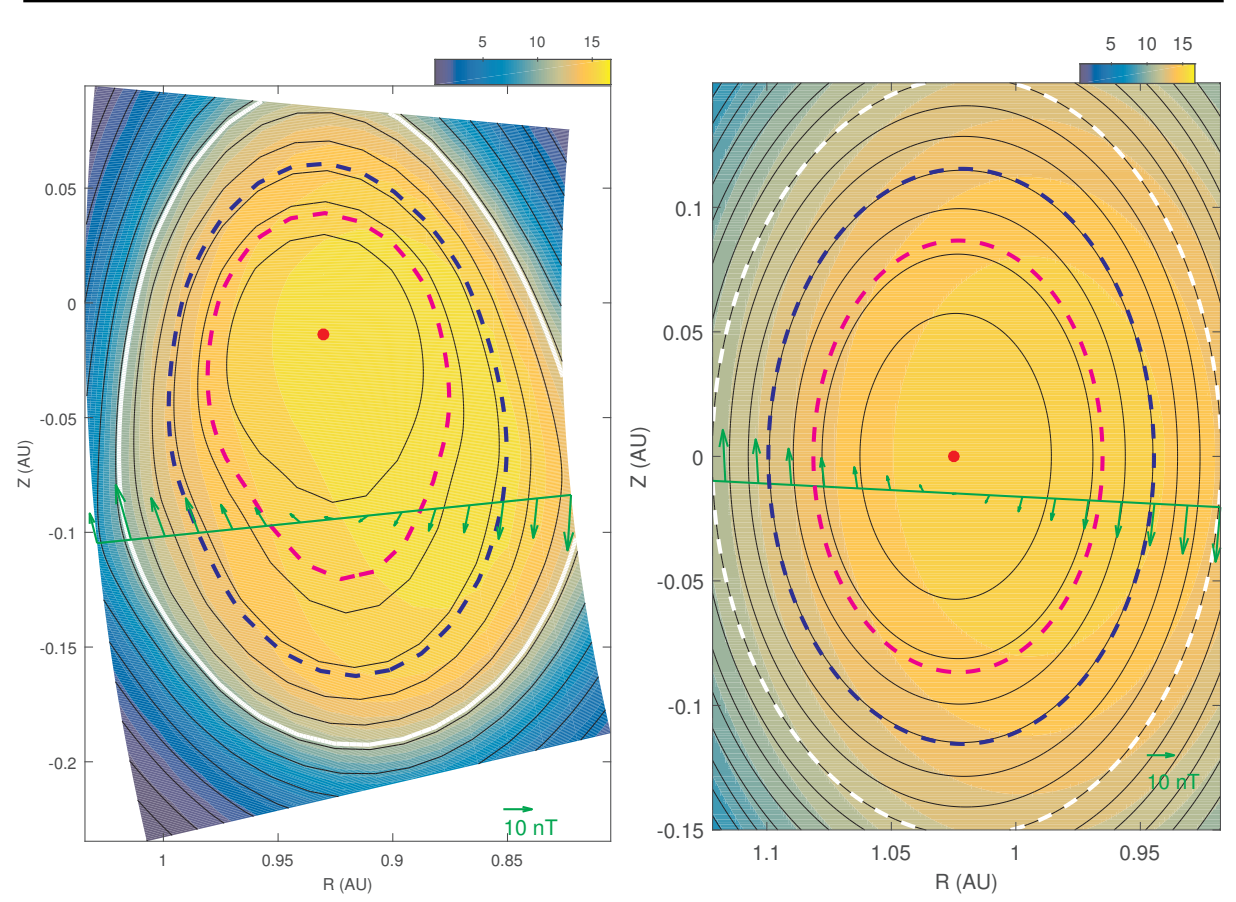

Figure 3. The resulting numerical GS solution (left panel) and the exact solution (right panel) for the benchmark case. In each panel, the black contour lines are equi-value contours of $\Psi$, and the filled color contour represents the axial field component $B_{\phi}$ with the same range of scales indicated by the colorbars (positive being out of the plane). The red dot and color dashed contours are the selected field lines of approximately the same $\Psi^{\prime}$ values, among which the red dot denotes the location where the transverse field vanishes. Additionally, the green arrows represent the measured transverse magnetic field components along the projected spacecraft path (the green line). The white contours represent $\Psi=0$ (dashed) and $\Psi=\Psi_{b}$, respectively.

on the $\left(r_{s c}, t\right)$ plane within certain domain as illustrated in Figure 1. The independent assessment of the goodness-of-fit is obtained by calculating the quantity $Q$ (Press et al., 2007, see also, Paper I), as shown in Figure 1p, associated with each search grid point. Such a quantity indicates the probability of a value drawn from a $\chi^{2}$ distribution greater than the specific $\chi^{2}$ value. Usually, a large $Q$ value close to 1 , together with reduced $\chi^{2} \lesssim 1$, indicates a "modestly" good fit (Press et al., 2007). We use the combined criteria of $\chi^{2} \leq 1$ and $Q \geq 0.9$ as the range of uncertainty for selecting the optimal parameters.

In this case, the regions of uncertainty given by both contours of $Q=0.9$ and $\chi^{2}=1$ nearly overlap on the $\left(r_{s c}, t\right)$ plane. We choose the location marked by the asterisk near the center of the closed contour $\chi^{2}=1$ as the optimal choice of the location of the $Z$ axis to carry out the final reconstruction procedures. The resulting major radius is $r_{0}=0.92 \mathrm{AU}$, which deviates from the true value, $r_{0}=1.02$ AU, by about $10 \%$.

Now we complete the last steps of the GS reconstruction by applying the GS solver described in Paper I to obtain a numerical solution of the GS equation, using the geometrical parameters determined above. These are additional steps not carried out in Paper I. All together, a complete real event study will start 

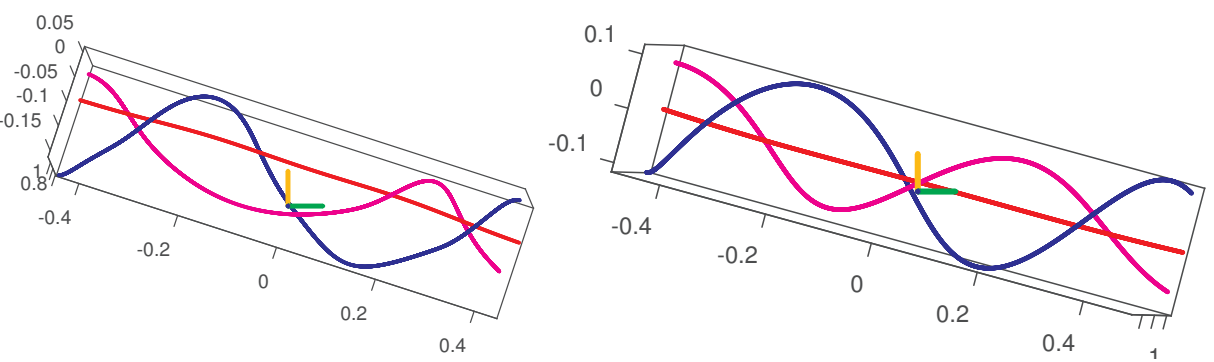

Figure 4. The $3 \mathrm{D}$ view against the radial direction, $r_{s c}$, of selected field lines of the same $\Psi^{\prime}$ values for both the numerical (left panel) and exact (right panel) GS solutions. The short thick lines are the $r_{s c}$ (blue), $t$ (green), and $n$ (gold) unit vectors, respectively, located at the point of spacecraft entry into the flux rope interval.
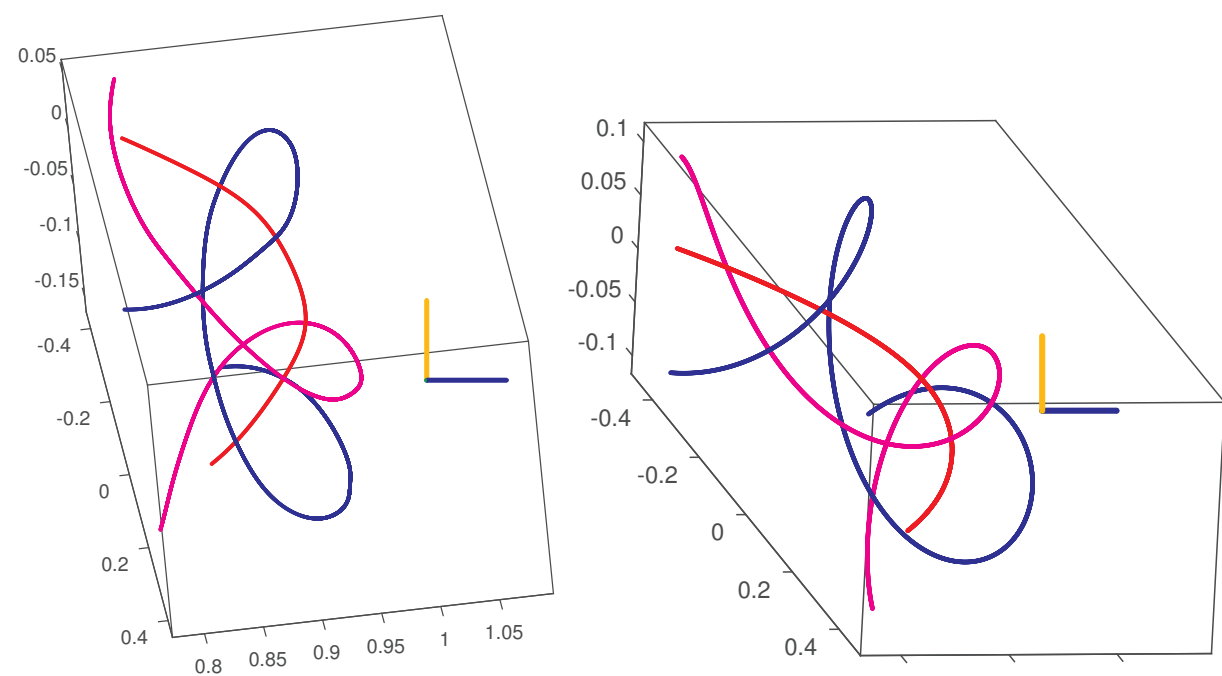

Figure 5. The solutions in $3 \mathrm{D}$ view along the direction $t$ with $n$ upward. The format is the same as in Figure 4

with the two-step recipe described in Paper I, followed by these last steps. Figure 2 shows the resulting $F$ versus $\Psi$ data plot along the spacecraft path and the corresponding fitting curve with a fitting residue $R_{f}=0.11$ defined as before (Hu et al., 2004). Figure 3 shows the cross sectional map of the numerical GS solution compared with the exact one. Since these two solutions are no longer lying on the same plane, due to different $Z$ axis orientations and different $r_{0}$ values, a quantitative evaluation of error through point-by-point subtraction is no longer valid. The two solutions clearly differ in overall shape, size and particularly the impact parameter, $d_{0}$, the shortest distance between the center of the flux rope (the red dot) and the projected spacecraft path (the green line). The overall axial field profiles are similar, but only within the boundary $\Psi=\Psi_{b}$ of the numerical solution and that $\Psi=0$ of the exact solution. Both are of lefthanded chirality. The axial field at the center is $16 \mathrm{nT}$ and $15 \mathrm{nT}$, respectively, for the numerical and exact solution. 

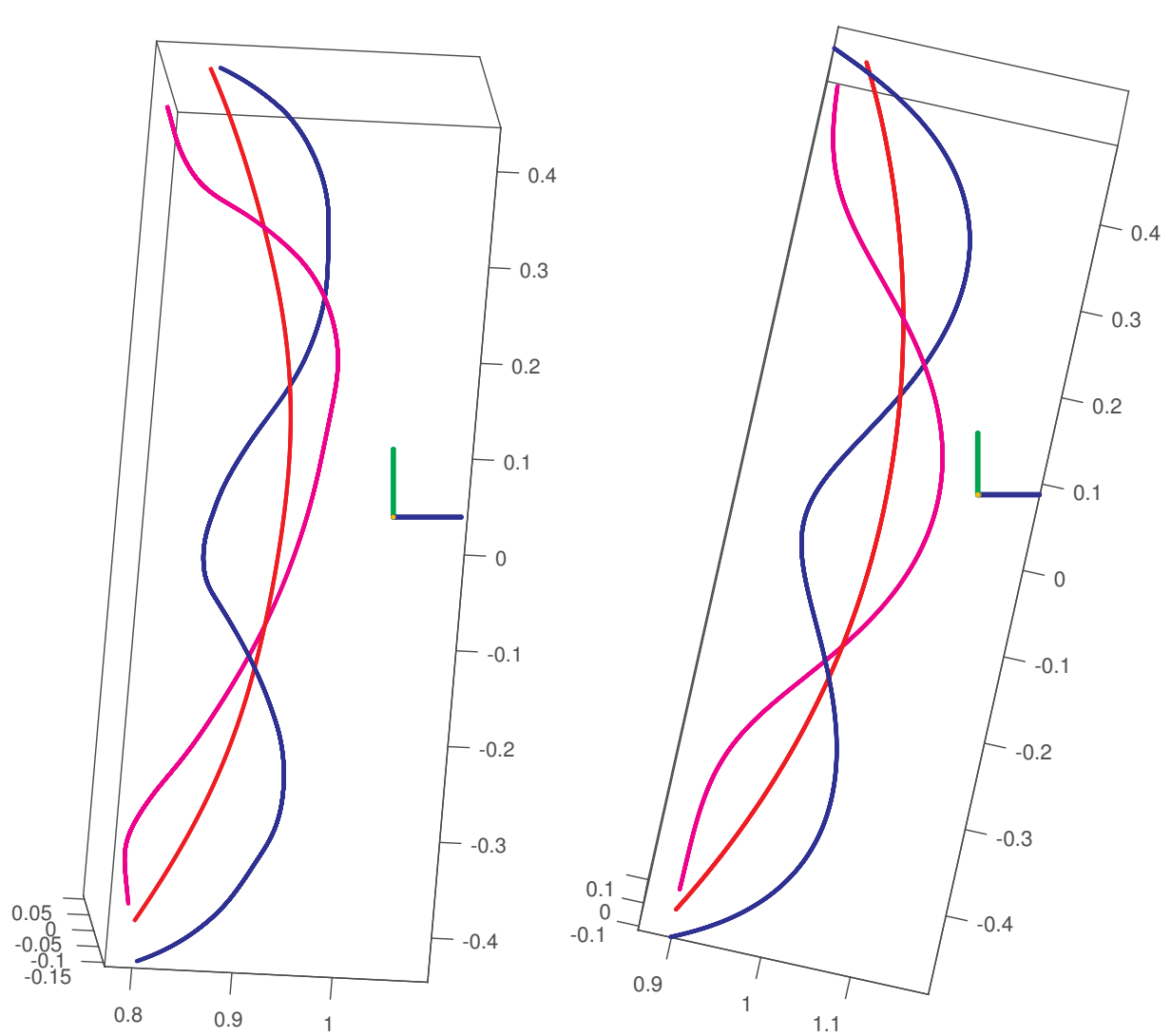

Figure 6. The solutions in $3 \mathrm{D}$ view against the direction $n$. The format is the same as in Figure 4

To further compare the magnetic field configuration between these two solutions, we present the 3D field-line plots of three selected field lines of similar $\Psi^{\prime}=\Psi-\Psi_{0}$ values, where the flux function value at the center is denoted $\Psi_{0}$, for both solutions. Their projections onto the cross sectional planes are shown in Figure 3 as nested closed loops by colored dashed lines including the red dots. They can be considered as the same set of field lines from the two solutions, thus can be inter-compared. Such comparisons are shown in Figures 4 , 5, and 6, for three different view angles, respectively. The discrepancies in their overall shapes are seen, albeit some field lines, for example, the central red and the outer blue ones appear to have better agreement between the numerical and the exact GS solutions.

In addition, we examine the quantitative output of some bulk properties derived from the GS reconstruction result. Table 1 summarizes the comparison of various output parameters between the numerical GS toroidal reconstruction and the exact solution. They include the central axial field $B_{\phi, 0}$, the poloidal and axial magnetic flux, $\Phi_{p, t}$, and the impact parameter $d_{0}$. Despite the large deviation in the impact parameter as we pointed out earlier, the other param- 
Table 1. Comparison of the outputs between the numerical $\left(R_{f}=0.11\right)$ and exact $\left(R_{f}=0.0\right)$ GS solutions.

\begin{tabular}{ccccc}
\hline$R_{f}$ & $B_{\phi, 0}(\mathrm{nT})$ & $\Phi_{p}\left(10^{12} \mathrm{~Wb} /\right.$ radian $)$ & $\Phi_{t}\left(10^{12} \mathrm{~Wb}\right)$ & $d_{0}(\mathrm{AU})$ \\
\hline 0.0 & 15 & 23 & 15 & 0.015 \\
0.11 & 16 & 23 & 14 & 0.080 \\
\hline
\end{tabular}

eters, including the magnetic flux content, agree between the numerical GS reconstruction result and the exact solution.

\section{Application to In Situ MC Events}

We present here a complete real MC event study by the toroidal GS reconstruction. The event was observed by the STEREO B (STB) spacecraft near 1 AU on 6-7 June 2008. This event was also examined by both the original straight-cylinder GS reconstruction, using the in situ STB data in Möstl et al. (2009), and by the white-light imaging reconstruction of the flux rope evolution through the inner heliosphere in Wood, Howard, and Socker (2010). Both studies focused on exploiting the remote-sensing observations and morphological modeling, which provides continuous coverage of CME propagation and morphology of the modeled flux rope structure en route from the Sun to 1 AU (see also, Wood et al., 2012). The current study contributes to the goal of linking remote-sensing observations with in situ measurements and modeling, especially in addressing the magnetic field configuration of the flux rope. For this particular event, an inter-comparison between these studies is available.

Figure 7 shows the time-series plot of both magnetic field and plasma parameters measured at STB. The GS reconstruction interval for the toroidal geometry is selected as marked by the vertical lines. This interval corresponds well to a region of depressed proton $\beta$ (electron temperature is not available) and elevated magnetic field magnitude. In addition, the speed and density profiles obtained from Wood et al.'s imaging reconstruction analysis are also superposed, indicating a good match in the density peaks enclosing the flux rope interval. These peaks correspond to the outer surface of the flux rope volume from whitelight imaging reconstruction, as visualized in Wood, Howard, and Socker (2010) (see also Figure 12 ).

The toroidal GS reconstruction is then performed, following the procedures laid out in Paper I and in the previous section. A standard set of plots and outputs is to be presented in a sequential order. Figure 8 displays the usual residue map, contours of residue, Res (see Paper I for definition), over a search grid of trial $Z$ axis. This results from the first step of the two-step recipe for determining the orientation of $Z$ through a trial-and-error process by enumerating all possible directions of $Z$. The thick black contour is of value, Res $=\min (R e s)+1$, representing the range of possible $Z$ axis orientations. Based on the recipe and the benchmark study in Paper I, the optimal $Z$ axis orientation is usually chosen 


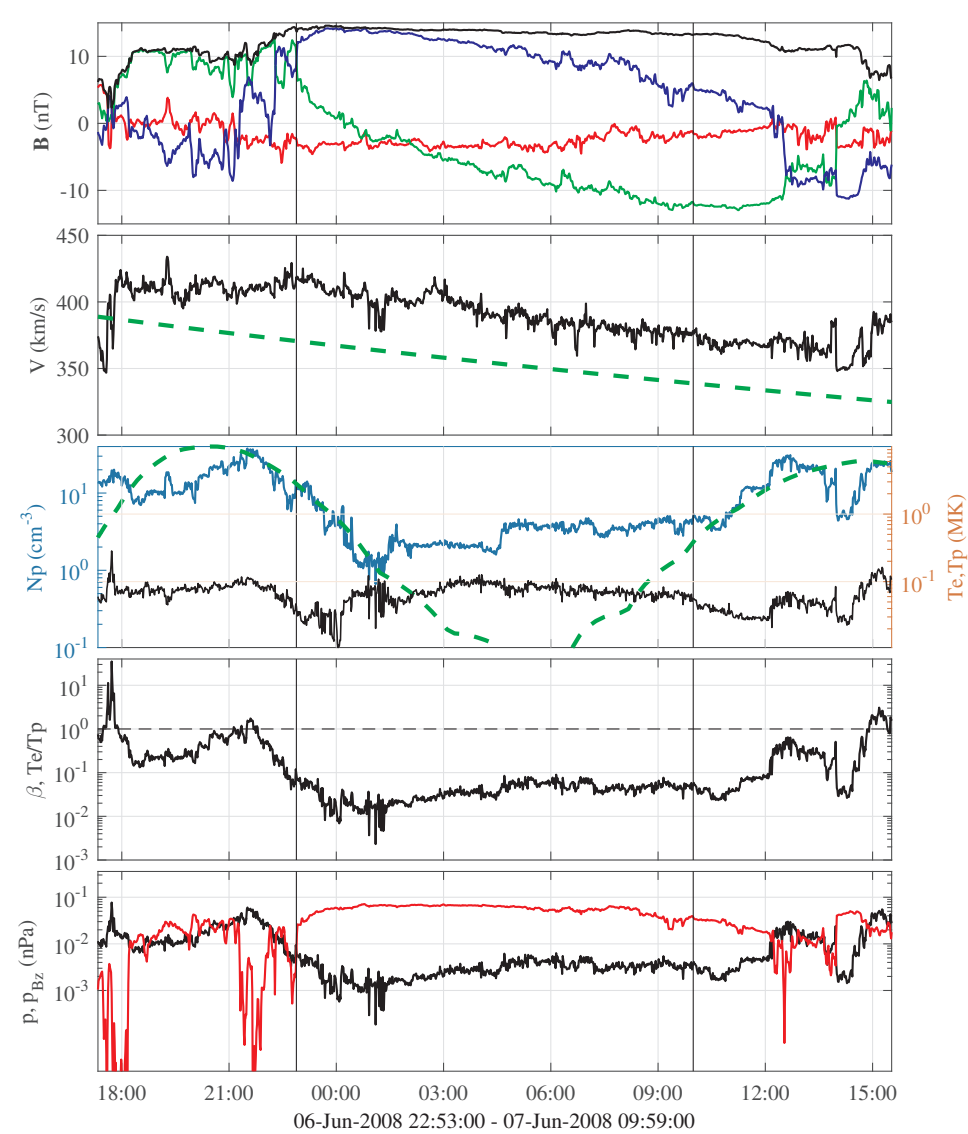

Figure 7. The time series from STB in situ measurements. From top to bottom panels: the magnetic field components in the spacecraft centered $\left(r_{s c}, t, n\right)$ coordinates and the magnitude in red, green, blue, and black, respectively, the solar wind speed, the proton density (left axis) and temperature (right axis; the electron temperature $T_{e}$ not available), the proton $\beta$ (the dashed horizontal line marks the value 1.0), and the plasma and axial magnetic pressure (red). The dashed green curves in the second and the third panels are the corresponding model predictions from Wood, Howard, and Socker $(2010)$. The vertical lines mark the GS reconstruction interval selected for this study, given beneath the plot.

as the point near the center of the contour, as marked by the cross sign, for such a singly connected contour. It is $Z=(0.5239,0.6626,0.5353)$ in the $\left(r_{s c}, t, n\right)$ coordinates and is used for the subsequent reconstruction. Optionally we also denote two other possible choices enclosed by the contour, which introduce significant uncertainty in the reconstruction result, but can be ruled out by other observations and associated analysis to be discussed later (see also Table 2 ).

Figure 9, in the same format as in Figure 1, demonstrates the determination of the other important geometrical parameter, the major radius $r_{0}$, or the intersection of the $Z$ axis with the $\left(r_{s c}, t\right)$ plane. This result is obtained from the second step of the two-step recipe of Paper I. Significant amount of uncertainty exists for the combined criteria of $\chi^{2} \leq 1$ and $Q \geq 0.9$ in determining an optimal solution of $r_{0}$. The range of uncertainty in $r_{0}$ is reflected by the area enclosed 


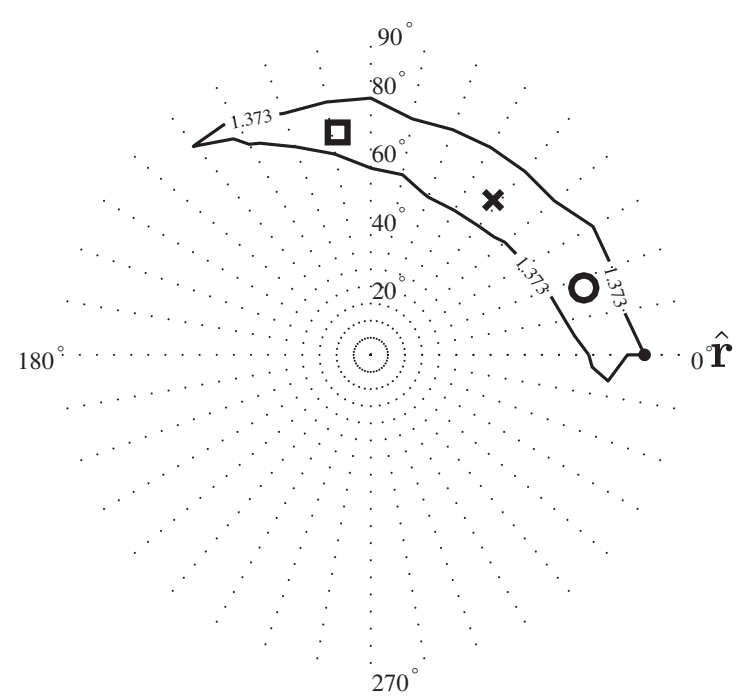

Figure 8. The residue map of the STB event: the contours of Res. The background dots are tips of unit vectors over the hemisphere of unit radius, enumerating all possible directions in space. The directions at 0 and 90 degrees correspond to unit vectors along $r_{s c}$ and $t$, respectively. The cross sign marks the optimal rotation axis $Z$ direction (see Paper I). The other two symbols mark alternatives inside the contour of value $1+\min ($ Res $)$.

(a)

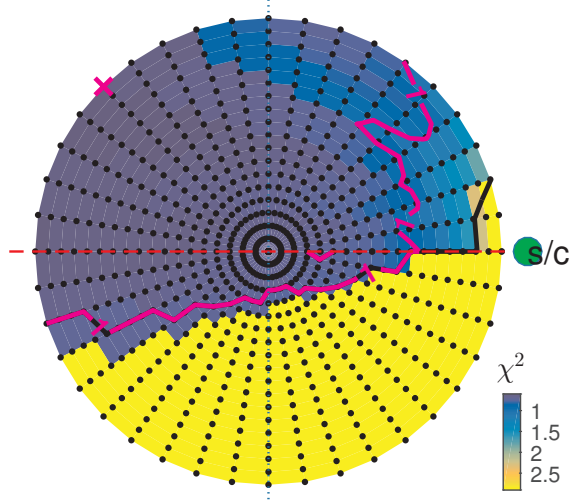

(b)

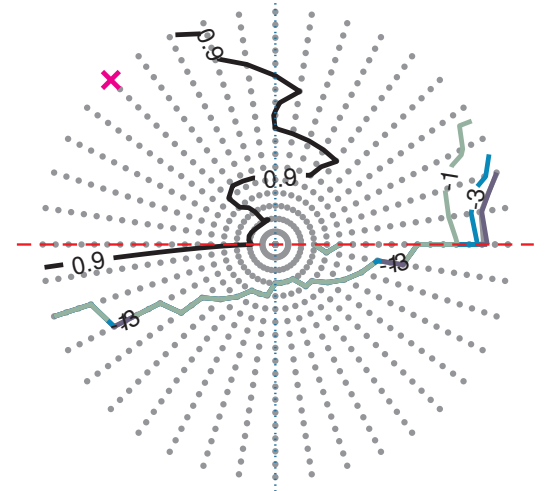

Figure 9. (a) The distribution of reduced $\chi^{2}$ value, as indicated by the colorbar on the $\left(r_{s c}, t\right)$ plane. (b) The corresponding contour plot of $\log _{10} Q$ as labeled except that the thick black contour is of level $Q=0.9$, and the outermost one is the same as the black one in (a). The format is the same as in Figure 1 The cross sign marks the location of $\chi_{\min }^{2}$

by the thick black contour in Figure 9b, which may extend to an even larger region beyond the search grid shown. For such a region enclosed by the $Q=0.9$ contour, the corresponding ranges of certain parameters including the major radius are given in Table 2. We choose the optimal $Z$ axis location at the point of minimum reduced $\chi^{2}$ value, $\chi^{2}=\chi_{\min }^{2}$, as marked by the magenta cross sign. This choice is considered representative, given that both the $\chi^{2}=1$ and $Q=0.9$ 


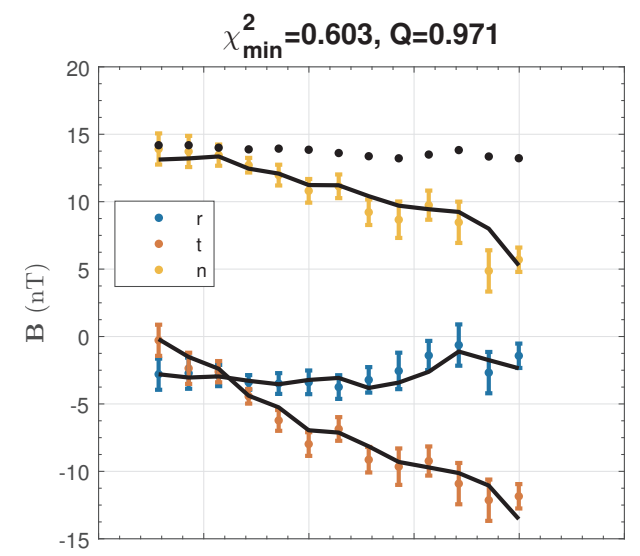

Figure 10. The magnetic field components (colored dots; see legend) along the spacecraft path with uncertainties (errorbars) and the corresponding GS model output (black lines) for the STB MC event. The associated minimum reduced $\chi^{2}$ and $Q$ values are given on top.

contour lines are open in this case. The resulting major radius is $r_{0}=1.70 \mathrm{AU}$. The corresponding reduced $\chi^{2}$ and $Q$ values are 0.603 and 0.971 , respectively, as indicated in Figure 10. There the measured magnetic field components and the output from GS model fitting of $F=R B_{\phi}$ versus $\Psi$ along the spacecraft path are shown, together with measurement uncertainties associated with each data point. Such uncertainties, $\sigma_{i}$, represented by errorbars associated with each point, are estimated from higher-resolution measurements by a calculation of the root-mean-squres of each magnetic field component $B_{\nu}$ within certain segment of $M$ data points with higher resolution, e.g. via $\left.\sigma_{\nu}^{2}=\sum_{i=1}^{M}\left\langle\left(B_{\nu i}-\left\langle B_{\nu}\right\rangle\right)^{2}\right\rangle\right]^{1}$. The measured quantities $F$ and $\Psi$ along the spacecraft path, and the corresponding fitting curve $F(\Psi)$ are given in Figure 11 . The final reconstructed cross section in an annular computation domain is shown in Figure 11 b, using the toroidal GS solver described and tested in Paper I.

The cross sectional map shows an elongated shape with fairly constant axial field, $B_{\phi}$, and a flux rope configuration of right-handed chirality. The center of the flux rope, defined as the location where the transverse field vanishes, locates at a distance $d_{0}=0.038 \mathrm{AU}$ away from the spacecraft path. At this location (the red point), the field line becomes "straight", i.e. it forms an exact circle around the $Z$ axis, concentric with the circle of major radius $r_{0}$. To further illustrate the configuration of such a toroidal flux rope, a 3D view with selected field lines is shown in Figure 12 (left panel) with a view angle radially toward the Sun from the STB point of view. The central field line in red is rooted on the red dot in Figure 11b, while the other two field lines are winding around this central axis of a finite curvature. Figure 12 (right panel), reproduced from Wood, Howard, and Socker (2010), shows the same view of the reconstructed flux rope from whitelight images, reaching STB's location at $1 \mathrm{AU}$. The colored surface represents the

${ }^{1}$ See the Ace Science Center: http://www.srl.caltech.edu/ACE/ASC/level2/mag_I2desc.html the calculation of the parameter, dBrms. 


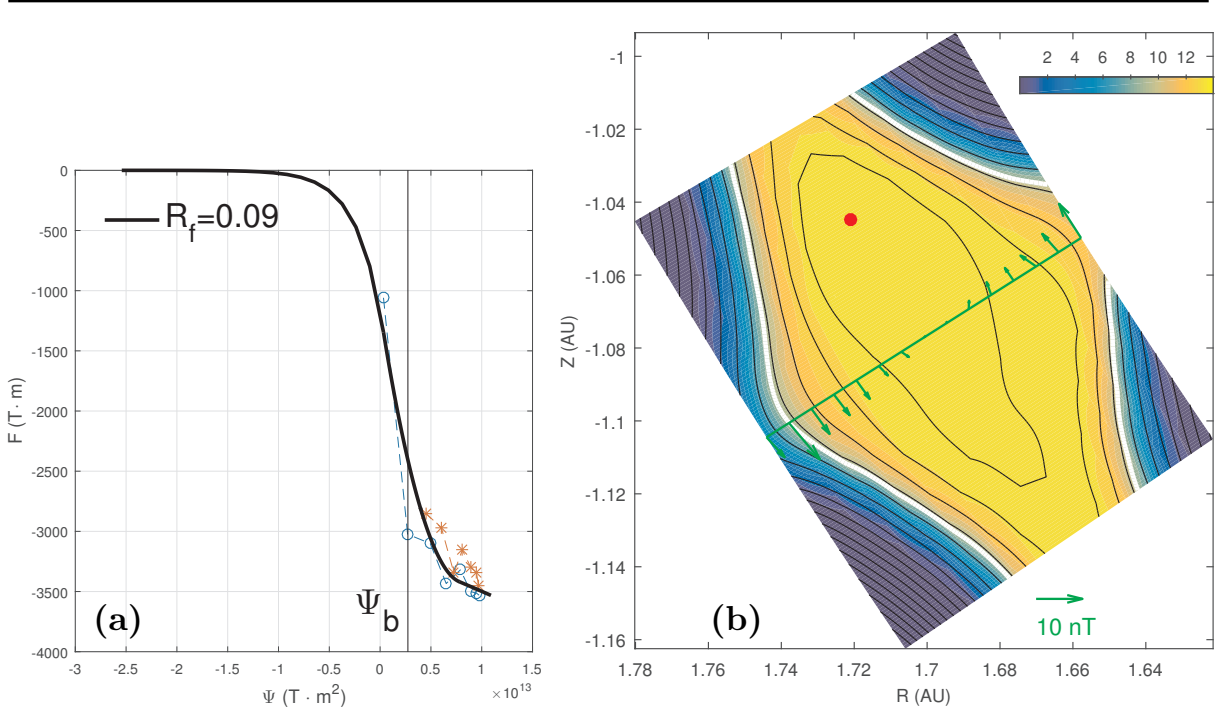

Figure 11. The toroidal GS reconstruction result for the STB MC event. (a) The composite function $F$ versus $\Psi$ and the fitting curve. The format is the same as in Figure 2 (b) The resulting cross section of the toroidal flux rope on the $(R, Z)$ plane. The format is the same as in Figure 3

boundary of the flux rope, corresponding to the peak density values in Figure 7. The reconstruction was validated by its success in reproducing the observed time or arrival at STB, and also its observed time of encounter with a comet that happened to be nearby (Comet Boattini). The inclination angle of the apparent central axis of the flux rope as seen away from the ecliptic plane is about 35 degrees (equivalent to a clock angle $\phi_{t}=145$ degrees, to be defined below).

Figure 13 attempts to illustrate the uncertainty in the geometry of the reconstructed toroidal flux rope configuration at the STB location due to the uncertainty in the location of the central rotation axis $Z$ while keeping its orientation fixed. The set of arcs represents the curvature of the toroidal flux rope at the point of spacecraft intersection. We define two angles associated with the directional vector tangent to each arc at the point of spacecraft location, namely, the cone angle $\phi_{r}$, the angle between the directional vector and the radial direction $r_{s c}$, in the range $[0,180]$ degrees, and the clock angle $\phi_{t}$, the angle of the projection of the tangent vector on the $(t, n)$ plane, measured from the positive $t$, in the range $[0,360]$ degrees. For this case $(Z$ chosen as the one marked by the cross sign in Figure 8), the ranges of these two angles are $\phi_{r} \in[90,107]$ degrees, and $\phi_{t} \in[118,128]$ degrees from the STB point of view toward the Sun (see Figure 12), with mean values 96 and 123 degrees, respectively. The supplementary angle to $\phi_{t}$ is in the range of $[52,62]$ degrees. Correspondingly, the ranges of major radius and poloidal flux are, $r_{0} \in[0.81,1.72] \mathrm{AU}$, and $\Phi_{p} \in[1.8,6.1] \mathrm{TWb}$, respectively.

The importance of the reconstruction of morphology of flux rope CMEs from the Sun to the spacecraft intersection by Wood, Howard, and Socker (2010) is 

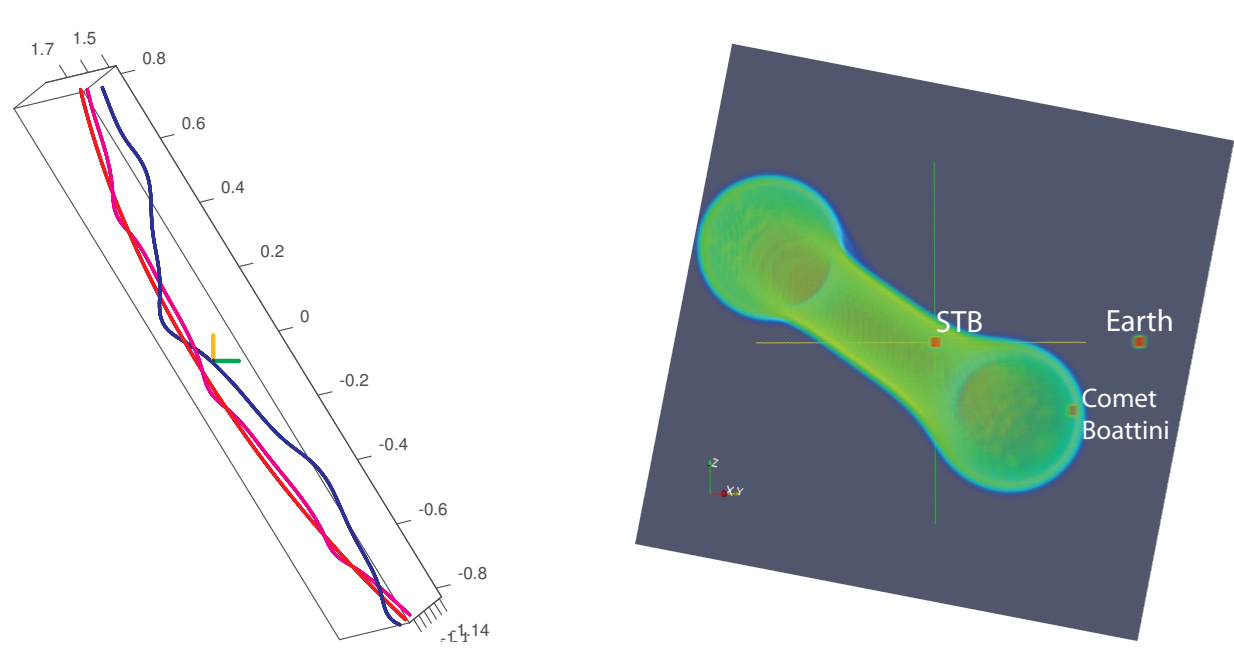

Figure 12. Left panel: the $3 \mathrm{D}$ view based on the reconstruction result of Figure 11 . The view point is toward the Sun. The format is the same as in Figure 4 Right panel: the flux rope configuration obtained from Wood, Howard, and Socker (2010) as seen from the same view angle and at the same location and time as the left panel. The STB location (the center dot) corresponds to the intersection of the short green and gold lines in the left panel.

Table 2. The ranges of parameter values for the three different choices of rotation axis orientations.

\begin{tabular}{ccccc}
\hline$Z\left(r_{s c}, t, n\right)$ & $r_{0}(\mathrm{AU})$ & $\Phi_{p}(\mathrm{TWb} /$ radian$)$ & $\phi_{r}$ (degrees) & $\phi_{t}$ (degrees) \\
\hline$(0.8663,0.2722,0.4188)$ & {$[0.77,1.27]$} & {$[2.3,4.8]$} & {$[101,105]$} & {$[118,125]$} \\
$(0.52390 .66260 .5353)$ & {$[0.81,1.72]$} & {$[1.8,6.1]$} & {$[90,107]$} & {$[118,128]$} \\
$(-0.13420 .90140 .4116)$ & {$[0.75,1.89]$} & {$[2.9,7.7]$} & {$[77,97]$} & {$[112,115]$} \\
\hline
\end{tabular}

that it provides guidance in reducing the uncertainty in determining the flux rope geometry when searching for the set of optimal parameters in the GS reconstruction. For instance, in this event, from Wood, Howard, and Socker (2010), the spacecraft crossed the flux rope above the center from the STB point of view toward the Sun (see Figure 12). Our analysis yielded the magnetic field configuration consistent with this above-the-center crossing for the set of optimal rotation axis $Z$ and the resulting major radius indicated above. Other choices of parameters, such as the alternative $Z$ axis orientations marked by the circle and the square in Figure 8 failed to yield such a configuration. In addition, the ranges of angles $\phi_{r}$ and $\phi_{t}$ are also more consistent with Wood, Howard, and Socker (2010) for the chosen optimal $Z$ axis in the middle of the residue contour in Figure 8, For completeness, the corresponding sets of parameters especially in terms of their ranges are summarized in Table 2 for the three choices of $Z$ axis orientations marked in Figure 8 in the order from the circle, cross to square symbols for rows 1-3.

A comparison with the corresponding GS reconstruction result from Möstl et al. (2009) with the straight-cylinder geometry shows certain similarities. Both 


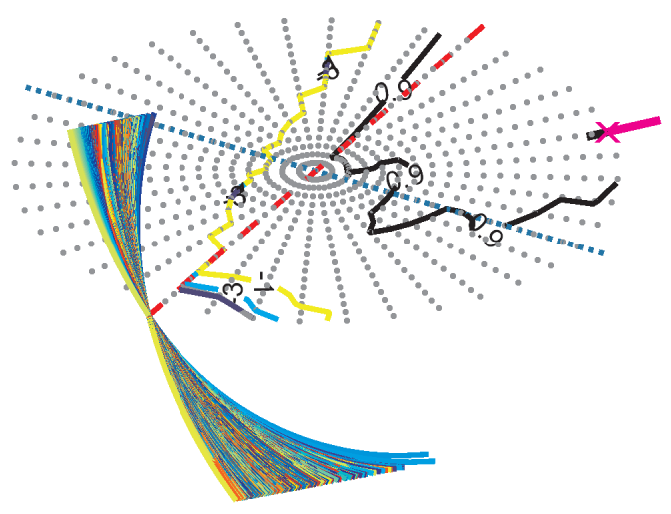

Figure 13. Figure 9p rendered in a different view angle. The red dashed line and the blue dotted line are the $r_{s c}$ and $t$ coordinates, respectively. Additionally, the thick pink line and the cross sign mark the direction and location of optimal $Z$ axis, the orientation of which is also marked on Figure 8 The bunch of arcs all crossing the spacecraft location at $r_{s c}=r_{s c 0}$ illustrate the sections of circles centered around the $Z$ axis whose location changes over the dots enclosed by the thick black contour $(Q=0.9)$ while maintaining its direction. Each colored arc is the result from the corresponding location in this case.

flux ropes are right-handed (see Figure 11). The axial field strength is close, 15.4 $\mathrm{nT}$ in Möstl et al. (2009) and $14 \mathrm{nT}$ from our analysis. The inclination of the axis of the flux rope with respect to the $\left(r_{s c}, t\right)$ plane is similar, $\sim 51$ degrees, comparable with our result, the mean value 57 degrees, for the axis lying nearly on the $(t, n)$ plane (Möstl et al., 2009). The axial (toroidal) and poloidal flux from Möstl et al. (2009) are 7.2 TWb and $11.9 \mathrm{TWb} / \mathrm{AU}$, respectively, noticeably larger than what we obtain in this analysis, $2.7 \mathrm{TWb}$ and $8.2 \mathrm{TWb} /$ radian, correspondingly, due to a smaller-size interval used in the current analysis and relatively large impact parameters in both analyses. 
The comparison of the inclination of the flux rope axis with respect to the $\left(r_{s c}, t\right)$ plane with Wood, Howard, and Socker (2010) shows a deviation of about 20-30 degrees. Our interpretation is that the GS reconstruction focuses on the magnetic field configuration embedded within what Wood et al. reconstructed, since the latter approach based on white-light images yields a density structure in terms of outer shells bounding the CME, enclosing the GS reconstruction interval in this case (see Figure 7). Therefore the magnetic flux rope from the GS reconstruction is largely enclosed by the dense, outer boundary of the CME flux rope, and they don't necessary share exactly the same apparent axial orientation although they should not differ dramatically, say, by 90 degrees, either.

\section{Conclusions and Discussion}

In conclusion, we have completed the development of the toroidal GS reconstruction method and conducted the first applications to both a benchmark study and a real MC event observed by STB. We provide an additional tool in modeling the magnetic flux rope configuration from in situ spacecraft data. The tool is ready for applications to additional events, using in situ spacecraft measurements and reasonable computing resources. The whole procedure can be completed on a desktop computer in Matlab within 2-3 hours. The benchmark study given here and in Paper I indicates that there is significant amount of uncertainty in recovering the exact geometry of the flux rope. In addition to deviations in the $Z$ axis orientation and the major radius (Paper I), the impact parameter, $d_{0}$, obtained from the final GS reconstruction of the cross section can differ most significantly from the exact value, 0.080 AU against $0.015 \mathrm{AU}$, in this study. Nonetheless, other physical properties, especially the magnetic flux, both the toroidal and poloidal component, are accurately recovered (see Table 1). We also apply the method to a real MC event measured by STB that was studied earlier by both in situ modeling by the straight-cylinder GS reconstruction and the white-light imaging reconstruction. The GS reconstruction result with the toroidal configuration is obtained with significant uncertainty. For the optimal rotation axis, $Z=(0.5239,0.6626,0.5353)$, chosen in the $\left(r_{s c}, t, n\right)$ coordinate system, the major radius is in the range [0.81, 1.72] AU. We compare the apparent geometry of the flux rope configuration with the flux rope volume resulting from white-light imaging reconstruction at the STB location. We find that the inclination angles of the central axis of the flux rope from the two results are generally consistent in terms of the ranges of the cone and clock angles of the axial direction. But the clock angle differs by about 20-30 degrees in the plane of the sky.

It is still not conclusive whether the toroidal geometry is superior to the original straight-cylinder geometry, despite the much evolved procedures in the toroidal GS reconstruction. The central axis orientation is similar at the point of spacecraft traversal. The magnetic flux content is very different, partially due to different time intervals used in these two separate analysis. For larger major radius, the two reconstruction results should converge. In other words, as the major radius $r_{0}$ goes to infinity, the curvature of the toroidal flux rope tends 
to zero, falling back to the original straight-cylinder analysis. This is somewhat hinted by the open contours in Figure 9 implying the possible consistency with a straight-cylinder geometry, for this case. The added feature in the current analysis, not yet available in the original straight-cylinder GS reconstruction is the uncertainty estimates in various quantities, based on proper $\chi^{2}$ statistics and the measurement uncertainties in magnetic field components. These are useful, but the caveat is that the results probably depend on a reliable estimate of the measurement uncertainties, which is not always available. Nonetheless, the unique scientific merit of the current fully developed approach lies in the ability to provide additional characterization of the magnetic flux rope variability in the space plasma environment.

Acknowledgments $\mathrm{QH}$ acknowledges partial support from NASA grants NNX14AF41G, NNX12AH50G, and NRL contract N00173-14-1-G006. All authors were supported as part of an FST team on flux ropes led by Dr. Linton and funded by NASA LWS award NNH14AX61I under ROSES NNH13ZDA001N.

Disclosure of Potential Conflicts of Interest The authors declare that they have no conflicts of interest.

\section{References}

Freidberg, J.P.: 2014, Ideal Magnetohydrodynamics, Plenum Press, New York, 196.

Hara, T., Seki, K., Hasegawa, H., Brain, D.A., Matsunaga, K., Saito, M.H.: 2014, The spatial structure of Martian magnetic flux ropes recovered by the Grad-Shafranov reconstruction technique. Journal of Geophysical Research (Space Physics) 119, 1262. DOI ADS

Hara, T., Luhmann, J.G., Halekas, J.S., Espley, J.R., Seki, K., Brain, D.A., Hasegawa, H., McFadden, J.P., Mitchell, D.L., Mazelle, C., Harada, Y., Livi, R., DiBraccio, G.A., Connerney, J.E.P., Andersson, L., Jakosky, B.M.: 2016, MAVEN observations of magnetic flux ropes with a strong field amplitude in the Martian magnetosheath during the ICME passage on 8 March 2015. Geophys. Res. Lett. 43, 4816. DOI ADS

Hau, L.-N., Sonnerup, B.U.Ö.: 1999, Two-dimensional coherent structures in the magnetopause: Recovery of static equilibria from single-spacecraft data. J. Geophys. Res. 104, 6899. DOI ADS

Hidalgo, M.A.: 2016, A Global Magnetic Topology Model for Magnetic Clouds. IV. Astrophys. J. 823, 3. DOI. ADS

Hu, Q.: 2017a, The Grad-Shafranov Reconstruction in Twenty Years: 1996 - 2016. Sci. China Earth Sciences in press. DOI. ADS

$\mathrm{Hu}$, Q.: 2017b, The Grad-Shafranov reconstruction of toroidal magnetic flux ropes: method development and benchmark studies. Solar Physics in press. DOI ADS

$\mathrm{Hu}$, Q., Sonnerup, B.U.Ö.: 2001, Reconstruction of magnetic flux ropes in the solar wind. Geophys. Res. Lett. 28, 467. DOI. ADS

Hu, Q., Sonnerup, B.U.Ö.: 2002, Reconstruction of magnetic clouds in the solar wind: Orientations and configurations. J. Geophys. Res. (Space Physics) 107, 1142. DOI ADS

Hu, Q., Qiu, J., Krucker, S.: 2015, Magnetic field-line lengths inside interplanetary magnetic flux ropes. J. Geophys. Res. 120, 1. DOI ADS

Hu, Q., Smith, C.W., Ness, N.F., Skoug, R.M.: 2004, Multiple flux rope magnetic ejecta in the solar wind. J. Geophys. Res. (Space Physics) 109, 3102. DOI ADS

Hu, Q., Qiu, J., Dasgupta, B., Khare, A., Webb, G.M.: 2014, Structures of Interplanetary Magnetic Flux Ropes and Comparison with Their Solar Sources. Astrophys. J. 793, 53. DOI. ADS

Kazachenko, M.D., Canfield, R.C., Longcope, D.W., Qiu, J.: 2012, Predictions of Energy and Helicity in Four Major Eruptive Solar Flares. Solar Phys. 277, 165. DOI ADS

Marubashi, K.: 1997, Interplanetary magnetic flux ropes and solar filaments. Washington DC American Geophysical Union Geophysical Monograph Series 99, 147. DOI ADS 
Marubashi, K., Akiyama, S., Yashiro, S., Gopalswamy, N., Cho, K.-S., Park, Y.-D.: 2015, Geometrical Relationship Between Interplanetary Flux Ropes and Their Solar Sources. Solar Phys. 290, 1371. DOI ADS

Möstl, C., Farrugia, C.J., Temmer, M., Miklenic, C., Veronig, A.M., Galvin, A.B., Leitner, M., Biernat, H.K.: 2009, Linking Remote Imagery of a Coronal Mass Ejection to Its In Situ Signatures at 1 AU. Astrophys. J. Lett. 705, L180. DOI ADS

Nieves-Chinchilla, T., Linton, M.G., Hidalgo, M.A., Vourlidas, A., Savani, N.P., Szabo, A., Farrugia, C., Yu, W.: 2016, A Circular-cylindrical Flux-rope Analytical Model for Magnetic Clouds. Astrophys. J. 823, 27. DOI ADS.

Press, W.H., Teukolsky, S.A., Vetterling, W.T., Flannery, B.P.: 2007, Numerical recipes in $\mathrm{C}_{+}+$: the art of scientific computing, 778, Cambridge Univ. Press, New York. DOI ADS

Qiu, J., Hu, Q., Howard, T.A., Yurchyshyn, V.B.: 2007, On the Magnetic Flux Budget in LowCorona Magnetic Reconnection and Interplanetary Coronal Mass Ejections. Astrophys. J. 659, 758. DOI ADS

Riley, P., Linker, J.A., Lionello, R., Mikić, Z., Odstrcil, D., Hidalgo, M.A., Cid, C., Hu, Q., Lepping, R.P., Lynch, B.J., Rees, A.: 2004, Fitting flux ropes to a global MHD solution: a comparison of techniques. J. Atmos. Solar-Terr. Phys. 66, 1321. DOI ADS

Romashets, E.P., Vandas, M.: 2003, Force-free field inside a toroidal magnetic cloud. Geophys. Res. Lett. 30, 2065. DOI. ADS

Sonnerup, B.U.Ö., Guo, M.: 1996, Magnetopause transects. Geophys. Res. Lett. 23, 3679. DOI ADS

Wood, B.E., Howard, R.A., Socker, D.G.: 2010, Reconstructing the Morphology of an Evolving Coronal Mass Ejection. Astrophys. J. 715, 1524. DOI ADS

Wood, B.E., Rouillard, A.P., Möstl, C., Battams, K., Savani, N.P., Marubashi, K., Howard, R.A., Socker, D.G.: 2012, Connecting Coronal Mass Ejections and Magnetic Clouds: A Case Study Using an Event from 22 June 2009. Solar Phys., 143. DOI ADS. 